\title{
MERLIN and eMERLIN OH maser observations toward the star forming region complex W49 A
}

\author{
Kitiyanee Asanok ${ }^{1}$, Sandra Etoka ${ }^{* 2}$, Malcolm Gray ${ }^{3}$, Anita Richards ${ }^{3}$; \\ Busaba-Hutawarakorn Kramer ${ }^{4,5}$, Nipon Gasiprong ${ }^{6}$ \\ ${ }^{1}$ Khon Kaen University, TH; ${ }^{2}$ Hamburger Sternwarte, DE; ${ }^{3}$ Jodrell Bank Centre for \\ Astrophysics, UK; ${ }^{4}$ Max-Planck-Institut für Radioastronomie, DE; ${ }^{5}$ National Astronomical \\ Research Institute of Thailand, TH; ${ }^{6}$ Ubon Ratchathani University, TH \\ E-mail: kitiyanee@kku.ac.th / sandra.etoka@googlemail.com
}

\begin{abstract}
We present the preliminary results from $\mathrm{OH} 18-\mathrm{cm}$ ground-state phase-referenced observations carried out with MERLIN (Multi-Element Radio Linked Interferometer Network) and e-MERLIN towards the star forming region (SFR) complex W49 A. There are three active SFRs in this complex: W49 North (W49 N), W49 South West (W49 SW) and W49 South (W49 S). The first epoch of observations was obtained in 2005 with MERLIN while the second epoch was obtained in 2013 with the e-MERLIN upgraded system. Here we present the 1612- 1665- and 1720-MHz maser emission towards W49 S and W49 SW. Overall, both MERLIN and e-MERLIN epochs show good agreement with the previous observations carried out with the VLA (Very Large Array). Due to a better sensitivity and a wider velocity coverage, we also found a new maser site in W49 S located $\sim 4.5$ arcsec south of the previously known site. Further analysis of the spatial distribution and kinematics of the masers in association with other disc and outflow tracers will allow us to get a clearer understanding of the morphology of this complex SFR region.
\end{abstract}

12th European VLBI Network Symposium and Users Meeting

7-10 October 2014

Cagliari, Italy

\footnotetext{
* Speaker.
} 


\section{Introduction}

$\mathrm{OH}$ masers have long been known to be associated with UC HII regions and bipolar molecular outflows from young stars, and are therefore recognised as signposts for active star formation and regions of extremely dense gas $\left(>10^{6} \mathrm{~cm}^{-3}\right.$, [3] and reference therein). Since maser transitions are inverted for a given range of physical conditions, combining different maser transitions even within the same species is a powerful probe of e.g. local density and temperature variations down to a few hundreds of AU. This combined information can help us distinguish between different possible physical structures (i.e., outflows or discs) around a given YSO [5], [2], [6].

The W49 complex can be found in the constellation of Aquila; it consists of a giant molecular cloud (GMC) W49 A to the west and a supernova remnant W49 B $\sim 12^{\prime}$ to the east. W49 A is one of the most massive star-forming regions in the Galaxy with a total mass of $\sim 10^{6} M_{\odot}$ within a radius of $60 \mathrm{pc}([7])$. It lies in the Galactic plane $\left(1=43 . .^{\circ} 17, \mathrm{~b}=+0 .{ }^{\circ} 00\right)$ at a distance of $11.11_{-0.69}^{+0.79} \mathrm{kpc}$ [9]. W49 A has 40 well studied UC HII regions associated with a minimum of 40 stars earlier than B3 [4]. It has three main far-infrared peaks; W49 N, W49 SE (so-called W49 S) and W49 SW [8].

\section{Observations and Results}

The phase-referenced observations of all ground-state $\mathrm{OH}$ maser lines towards W49 A were carried out with MERLIN in 2005 and the e-MERLIN upgraded system in 2013. Both epochs used the same telescopes, namely: Defford, Cambridge, Knockin, Darnall, Mark 2 and Pickmere/Tabley. For the MERLIN 2005 observations, the phase-reference calibrator 1922+086(J2000) was used. 3C286 and 3C84 were used as flux and bandpass calibrators, respectively. For the e-MERLIN 2013 observations, the same phase-reference calibrator (i.e., 1922+086(J2000)) was used. 1407+284 (or OQ208) was used to set the flux scale, its flux density having previously been derived from 3C286. 3C84 was used for bandpass calibration. Both epochs have an absolute positional accuracy of 0.05 arcsec. Here we present the preliminary results from the $1612-\mathrm{MHz}, 1665-\mathrm{MHz}$ and $1720-$ $\mathrm{MHz}$ maser lines in W49 S and W49 SW.

The final-datasets spectral profiles and amplitudes observed at both the MERLIN and eMERLIN epochs are similar for the 1665 and 1720-MHz maser lines for both W49 S and W49 SW. The 1720-MHz OH masers have a velocity spread of less than $5 \mathrm{~km} \mathrm{~s}^{-1}$ in both regions, while the $1665-\mathrm{MHz} \mathrm{OH}$ masers have velocities ranging from $\sim+5$ to $+25 \mathrm{~km} \mathrm{~s}^{-1}$ in $\mathrm{W} 49 \mathrm{~S}$ and $\sim+2$ to $+16 \mathrm{~km} \mathrm{~s}^{-1}$ towards W49 SW. For the 1612-MHz spectra, however, there is a change in the spectral profile and velocity coverage observed toward W49 S. In the 2005 MERLIN epoch, the spectral velocity ranges from $\sim+12$ to $\sim+21 \mathrm{~km} \mathrm{~s}^{-1}$ while it ranges from $\sim+10$ to $\sim+18 \mathrm{~km} \mathrm{~s}^{-1}$ at the 2013 e-MERLIN epoch.

The left panel of Figure 1 presents the spatial distributions of the 1720-MHz maser components in W49 S for the MERLIN and e-MERLIN epochs as well as the maser component distribution from previous VLA observations obtained by [1] which have an absolute positional accuracy of $0.3 \times 0.5 \operatorname{arcsec}^{2}$. Note that the VLA and (e)MERLIN positional accuracies are displayed by 

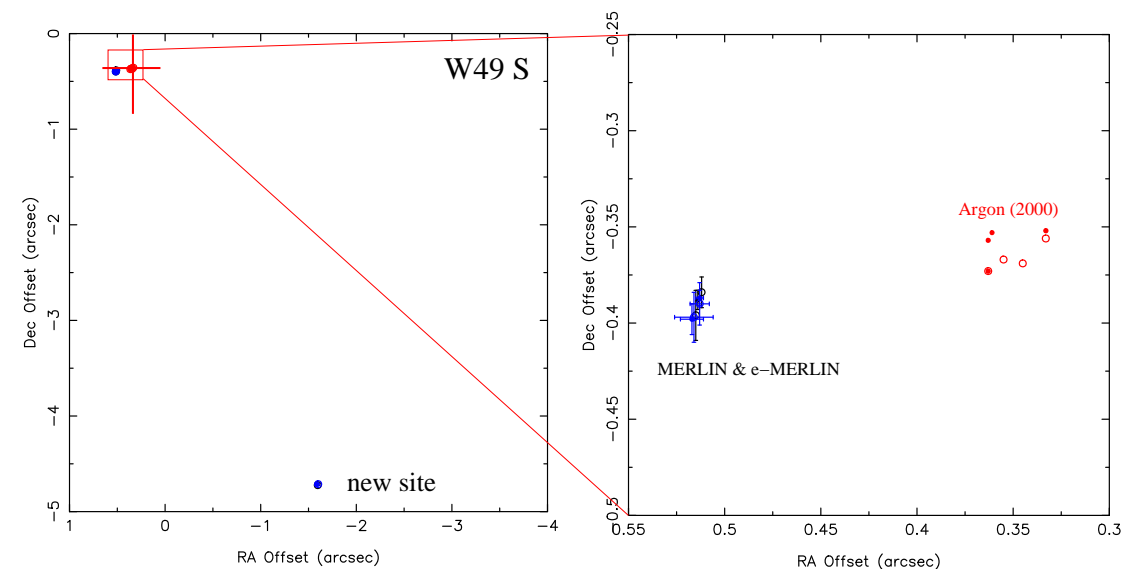

Figure 1: The position comparison of the 1720$\mathrm{MHz}$ masers in W49 $\mathrm{S}$ as measured by MERLIN (in blue), e-MERLIN (in black), and VLA (in red, from [1]). The offset $(0,0)$ is $\mathrm{RA}=19^{h}$ $10^{m} 21^{s} 65$ Dec. $=+09^{\circ} 05^{\prime}$ 02." 6 . Solid and open symbols are left-hand circular polarisation (LHC) and right-hand circular polarisation (RHC) respectively.

the crosses in the left and right panels, respectively. The MERLIN and e-MERLIN distributions are in good agreement. The northern group position agrees with the group detected by [1] within their positional accuracy (right-hand side of Figure 1). We discovered a new 1720-MHz maser site (detected by MERLIN and confirmed by e-MERLIN; see left-hand side of Figure 1) located $\sim 4.5$ arcsec south of the previously known site. This group of masers has velocities ranging from $\sim+12.98$ to $\sim+13.53 \mathrm{~km} \mathrm{~s}^{-1}$ and has a peak flux density $\sim 0.21 \mathrm{Jy} \mathrm{beam}^{-1}$.
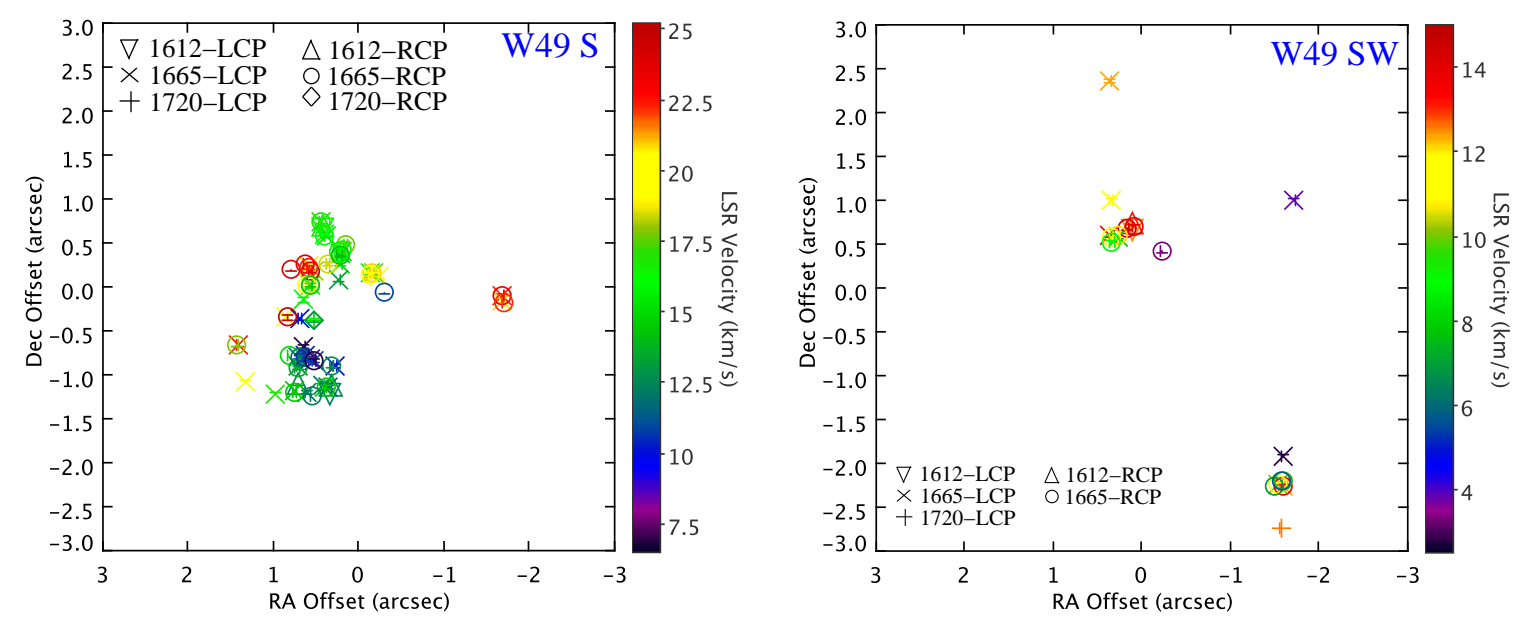

Figure 2: Relative-position and velocity distributions of the 1612-, 1665- and 1720-MHz masers in W49 S (left) and W49 SW (right) from the e-MERLIN observations. For the offset $(0,0)$ in W49 S cf. Fig. 1. The offset $(0,0)$ for W49 SW is RA $=19^{h} 10^{m} 11 .^{s} 04 \mathrm{Dec}=+09^{\circ} 05^{\prime} 20 .^{\prime \prime} 2$.

The spatial and velocity distributions of the e-MERLIN OH masers in W49 S and W49 SW are displayed in Figure 2 (left- and right-hand sides, respectively). In both regions, the overall spatial distributions are within $\sim 6^{\prime \prime} \times 6^{\prime \prime}$ which is equivalent to $\sim 0.3 \times 0.3 \mathrm{pc}^{2}$ (at the distance given by [9]). In W49 S, the bulk of the emission is confined in a rather compact "C-shaped" 
region. On the other hand, in W49 SW, the bulk of the emission traces an NE-SW elongated structure. Both sources show complex maser velocity distributions. A further analysis, with e.g. additional existing outflow and disc molecular-tracer data, should help us to understand the nature of the complex velocity distributions observed in these regions.

\section{Conclusions}

We have presented preliminary results from phase-referenced observations of the 1612-, 1665and 1720-MHz OH masers in W49 S and W49 SW obtained with MERLIN (in 2005) and eMERLIN (in 2013). Both position and velocity distributions of the masers from both epochs of observations are consistent within the positional uncertainties. For the previously detected VLA sites and taking into account their positional accuracy, our maser positions agree well. We discovered a new 1720-MHz OH maser site in the south part of W49 S. The overall spatial distributions and kinematics of the $\mathrm{OH}$ masers is quite complex and will be subsequently studied together with other (outflow and disc) molecular tracers associated with the star-formation process already identified in these regions. This will allow us to better constrain the physical and dynamical properties of these regions. The data analysis of the maser emission towards $\mathrm{W} 49 \mathrm{~N}$ as well as the $1667 \mathrm{MHz}$ maser emission towards W49 S and W49 SW are currently a work in progress. Their analysis will allow us to draw an overall picture of the OH maser emission present in the W49 A SFR complex.

\section{Acknowledgments}

MERLIN is a National Facility operated by the University of Manchester at Jodrell Bank Observatory on behalf of STFC. KA would like to thank Thailand Research Foundation \& National Astronomical Research Institute of Thailand (NARIT) for partial financial support.

\section{References}

[1] Argon, A.L., Reid, M.J., \& Menten, K.M. 2000, ApJS, 129, 159

[2] Asanok K., Etoka S., Gray M.D., Thomasson P., Richards A.M.S., Kramer B.-Hutawarakorn, 2010, MNRAS, 404, 120

[3] Cohen, R.J. 1989, Reports on Progress in Physics, 52, 881

[4] De Pree, C.G., Mehringer, D.M., \& Goss, W.M. 1997, ApJ, 482, 307

[5] Etoka S., Cohen R.J. \& Gray M.D., 2005, MNRAS, 360, 1162

[6] Etoka S., Gray M.D. \& Fuller G.A., 2012, MNRAS, 423, 647

[7] Galván-Madrid, R., Liu, H.B., Zhang, Z.-Y., et al. 2013, ApJ, 779, 121

[8] Harvey, P.M., Campbell, M.F., \& Hoffmann, W.F. 1977, ApJ, 211, 786

[9] Zhang, B., Reid, M.J., Menten, K.M., Zheng, X.W., Brunthaler, A., Dame, T.M., \& Xu, Y. 2013, ApJ, 775,79 